\title{
Research on Tai Chi Tourism Market Development
}

\author{
Qiu Xueqin \\ School of Business Administration and Tourism \\ Management \\ Yunnan University \\ Sri Lestari \\ School of Business Administration and Tourism \\ Management \\ Yunnan University
}

\author{
Wan Hong \\ School of Business Administration and Tourism \\ Management \\ Yunnan University \\ Ilan Mukusheva \\ School of Business Administration and Tourism \\ Management \\ Yunnan University
}

\author{
Liu Zeshi \\ School of School of Public Administration \\ Yunnan University
}

\begin{abstract}
Tai Chi is a combination of body building, selfcultivation and martial arts. It has been widely spread both internationally and domestically. The main purposes of Tai Chi practitioners are learning Tai $\mathrm{Chi}$ and joining Tai Chi competitions. Meanwhile, the non Tai Chi practitioners are attracted by Tai Chi, leaving the place of residence to different places and take sightseeing activities, and thus the Tai Chi tourism is produced. This paper analyzes the development status, existing problems and market development strategies of Tai Chi tourism.
\end{abstract}

Keywords_Tai Chi; tourism; market development; strategies

\section{The Current Development of TAi Chi Tourism}

\section{A. Tai Chi Sightseeing Tour}

Many martial arts schools in China are inhabited in famous mountains and named after these mountains, such as Wudang school, Emei school and Qingcheng school, which have beautiful natural scenery and historical and cultural accumulation. Many tourists who come for the martial arts schools, while visiting famous teachers, are also enjoying the natural scenery and historical sites. The earliest birthplace of Tai Chi is in Wudang Mountains, which is a famous mountain with beautiful scenery. The students who were learning Tai Chi in Wudang Mountains, were also the tourists there. According to statistics, from 1990s to 2014, there were about 7000 foreigners visiting Wudang Mountains that were learning Tai Chi for more than 3 months, and even more for those who were learning Tai Chi for less than 3 months. Data presentation shows that in 2017, Wudang Mountains received 8 million and 881 thousand international tourists, and taking in tourism revenue of CNY 5 billion and 250 million, an increase of $16.75 \%$ and $22.09 \%$ over the same period of the last year. Students who are learning Tai Chi in Chen Jia Gou, Henan often have to travel to Luoyang, Kaifeng, Songshan and other famous tourist attractions in Henan. In this way, places surrounding the Tai Chi activities have become Tai Chi sightseeing tourism attractions.

\section{B. Tai Chi Competition Tour}

China has held several international and domestic Tai Chi events, such as the Wudang Mountains in Hubei, Jiaozuo in Henan, and Yongnian in Hebei. Since 1992, in Wenxian County, Jiaozuo City, Henan, China began to hold the international Tai Chi annual meeting, taking Tai Chi as the point of penetration, setting up a bridge of Tai Chi foreign exchange, and after that the event was hold once in every two years, attracting more than 100 thousand tourists. In 2015, the Eighth Jiaozuo International Tai Chi exchange competition was held together with the 2015 Yuntai Mountain Tourism Festival at the same time. Tai Chi was used as the medium, and the scenery was the mediator, having the theme of "Jiaozuo - The Holy Land of Tai Chi". During the competition, 23 cultural tourism projects in Jiaozuo were settled, with a total investment of CNY 12 billion 970 million, including the health tourism of Hainan Airlines, the large real performance of Yuntai Mountain in Xiu Wu County, the International Ecotourism resort of Miao Le temple tower in Wuzhi County, and the construction project of Dan River Canyon culture and tourism complex in Qinyang city. This Tourism Festival is directed by the National Tourism Administration and the United Nations World Tourism Organization, Monitoring Center of Sustainable Tourism Organization (MCSTO), the Henan Tourism Bureau and the Government, Tourism Bureau of Jiaozuo, the Government of Xiu Wu County and the Yuntai Mountain scenic Area Administration Bureau. During the Tai Chi competition, while also the Tourism Festival, 10 series of themes were held: the Jiaozuo Observatory Monitoring Activities of the Henan Observatory for Sustainable Development of the United Nations' World Tourism Organization, "Meet in Yuntai", China's Famous Mountain and Henan Tourism Product Exhibition, the launching ceremony of "The Publicity and Education Week in Henan 
and Jiaozuo Observatory of United Nations' World Tourism Organization Tourism Sustainable Development"; " Meet in Yuntai ", China's Famous Mountain and Henan Tourism Products Exposition; "Leisure in Yuntai", International Symposium on Sustainable Development of Mountain Tourism in China; International and Domestic Visitors Examining the Activities of Tourism Products in Henan; Tai Chi enthusiasts Seeking Ancestor Roots Activities In Wenxian County; Qingtian River Camping Festival; Mountain-climbing Competition in Shennong Mountain; and Jia Ying Guan Cultural Tourism Festival. The holding of Tai Chi events has played a positive role in promoting the development of local tourism. The Ninth China Jiaozuo International Tai Chi Exchange Competition held in 2017 was named "The Ninth China Jiaozuo International Tai Chi Exchange Competition and The 2017 Yuntai Mountain Tourism Festival", jointly sponsored by the National Sports Administration's martial arts sports management center, the Chinese Wushu Association, the Henan Provincial Sports Bureau, the Henan Provincial Tourism Bureau and the government of Jiaozuo. More than 3500 athletes from more than 50 countries and regions have participated in a number of competitions, such as the martial arts skills and tricks, instruments, hand pushing and many other events. During the period, a series of activities related to the sustainable development of the International Mountain Tourism in Jiaozuo, The International Famous Mountain and the "China Source" Henan Tourism Product Exhibition Week, the Yuntai Mountain International Scenery Photography Exhibition and other tourism related activities were held.

\section{Tai Chi Study Tour}

Tai Chi practitioners go far to seek teachers in learning Tai Chi, thus promoting the development of Tai Chi study tour. The writer has personally visited the Chen Jiagou for an observation, taking Chen Jiagou International Tai Chi Academy as an example, there are students from Britain, the United States, Germany, Japan and other parts. Students from other provinces in China take a break every ten days. They will arrange tours to Shaolin Temple, Luoyang, Kaifeng and Anyang. Many students think that a combination of doing tour and learning martial arts is better than pure sightseeing. The common sightseeing tour brings back some pictures and memories, but martial art tourism can also bring back some "Kungfu". It is beneficial for them because they can exercise or defend themselves. In Chen Jiagou International Tai Chi Academy, there are no lack of teenagers and four or five years old children who have been brought to practice Tai Chi by their parents. Parents take part in adult classes, and children take part in children's classes or teenager's classes.

\section{Tai Chi Seeking Ancestor Roots Tour}

Most of the practitioners who love certain martial arts sport want to visit the origin of this martial arts sport, which produces the motive of tourism. Worshipping Zhang Sanfeng, a Tai Chi Master in Wudang Mountains; finding ancestors when visiting Chen Jiagou, have become the purpose of many practitioners to travel outside. Chen Xiaowang, the Chen Style Tai Chi founder who lived in Australia would take his foreign students to the ancestral temple of the Chen Jiagou every year to worship the ancestors. These activities provided an opportunity for the development of tourism. On August 6th August 8th, 2017, more than 50 people from Europe (UK, Italy, Portugal), with their devotion and respect for Tai Chi, went to Zhengzhou, China, and went to the birthplace of Chen's Tai Chi, visited the master Chen Zhenglei. While learning Tai Chi, they also visited the Chen Jiagou, sought roots and respected the ancestors. The 148 royal court Tai Chi disciples in Ya'an, Sichuan, visited the tomb of Master Zhang Jinling in Kunming, Yunnan, and Master Zhang Jinling moved from Beijing to Kunming in Yunnan during the war of resistance in 1939. The 148 royal court Tai Chi disciples in Foshan, Guangdong, are also preparing to master the 148 imperial court Tai Chi art from Master Zhang Guoxiang in Kunming, Yunnan.

\section{The Existing Problems in the Tourism Development OF TAI CHI}

\section{A. Insufficient understanding of the potential of Tai Chi tourism development}

Tai Chi, with 300 million practitioners worldwide, is the most widely used fitness and regimen in the world. If such a large group of practitioners is developed into a combination of tourism and vacation, tourism and health preservation, it will have broad market prospects. Most government departments and enterprises have not yet seen the potential of this subdivision market. Although the Henan province has done well in this area, the coverage is far from enough, especially the lack of concrete and feasible project planning and design.

\section{B. Lack of organizations from tourism department.}

Tai Chi is a sport, and the Tai Chi competition is generally approved and managed by the sports department. This leads to the difficulty of ensuring the source of the ticket in the organization of large Tai Chi competition. Even in the event of a large competition, the tourism department cannot receive information or receive incomplete information. As a result, a large number of tourists come in and the scenic spots are not prepared enough, arrangements are ineffective, missing business opportunities and causing complaints. The Eighth and the Ninth International Tai Chi exchange competitions held in Jiaozuo, Henan, which are directly involved in the organization and management of tourism management departments, are worthy of promotion.

\section{Lack of travel agency participation}

To build Tai Chi tourism into a tourist product with characteristics of China and international influence, it is not enough to depend solely on the sports department and the martial arts department. The participation of tourism departments, especially the travel agencies is very important. The development and design of tourism products is one of the main duties of travel agencies. In particular, the major travel agencies need to go abroad to sell special tourism products every year. Some big travel agencies and foreign institutions have to fully mobilize travel agencies to package Tai Chi tourism products and sell them using convenient sales channels, so that they can bring in more visitors. 


\section{The Development Prospects of TAi Chi Tourism MARKET}

\section{A. Great potential for the development of Tai Chi tourism market}

Currently Tai Chi has spread to more than 150 countries and regions. There are 300 million Tai Chi practitioners in the world. Tai Chi organizations have been established in more than 80 countries and regions. Tai Chi is also known as the world's first well-known martial arts.

"Two boxing" (Shaolin boxing, Tai Chi) is the "fist" product of Henan tourism. In order to highlight the advantages of "two boxing", Henan tourism has made "Kungfu Tour" brand. In terms of Tai Chi study tour and Tai Chi event tourism, Henan province has been in the forefront. Wudang Mountains's Tai Chi study tour and Tai Chi event tourism is also doing well. As the birthplace of Yang style and Tai Chi martial arts, Yongnian, Hebei, also regularly held international Tai Chi competitions, attracting large numbers of competitors and tourists domestically and internationally. The Lijiang International Traditional Wushu Festival, held every two years in Lijiang, Yunnan province, also attracts a large number of Tai Chi enthusiasts at home and abroad to participate in the competition and sightseeing. The international Tai Chi competition held annually in Hong Kong attracts a large number of mainland practitioners to participate in and travel to Hong Kong.

Because the tourists have the psychological needs of seeking new and distinctive experiences, it is difficult to attract the same group of Tai Chi practitioners with the main purpose of tourism in the same city. This requires the development of a new Tai Chi competition city to activate the needs of such competitors. But at the same time, Tai Chi event held in the same city can attract new participants for the competition who are also taking part in sightseeing activities.

\section{B. Establishing new Tai Chi tourism hot cities under The One Belt and Road initiative}

Whether it is Hubei Wudang Mountains, Henan Chen Jiagou or Hebei Yongnian, it has become the main reason for Tai Chi study tour tourism, the ancestor seeking tourism and the event tourism. These places are the birthplace of Wudang Tai Chi, Chen Style Tai Chi, Yang style and martial Style Tai Chi. In addition to the origin of Tai Chi; can we further excavate the heritage and culture of Tai Chi to form a new Tai Chi tourist hot city? The writer believes that it should be possible through research and excavation, government leadership and participation from all walks of life.

Xi'an, as one of the four ancient capitals of the world, has a history of more than 3,000 years and a history of capital construction for 1100 years. It is the starting point of the ancient Silk Road. In Xi'an, Chen Style Tai Chi and Yang Style Tai Chi have a wide range of practitioners and are one of the important cities for the spread of Tai Chi in western China. As the ancient capital of the sixteen dynasties, Xi'an has a wealth of historical and cultural tourism resources and is very suitable for Tai Chi tourism.
The second one is Yunnan. Yunnan is an important strategic location in the national "One Belt and One Road" strategy. It is an important strategic node and portal. The north of Yunnan can connect with the Silk Road Economic Belt and the south connects with the Maritime Silk Road. It is the only province in China that can simultaneously link the land and sea together with Southeast Asia and South Asia nations. Yunnan has 16 first-class ports and 7 second-class ports, bordering Myanmar, Vietnam, and Laos. It is also close to Malaysia, Singapore, India, and Bangladesh. It is one of the provinces with the largest number of neighboring countries. When President Xi Jinping visited Yunnan, he pointed out that Yunnan should be built to become a radiation center for Southeast Asia and South Asia.

Yunnan Province not only has abundant tourism resources of natural and ethnic customs. Kunming, the provincial capital's climate in February and March is having a lot of flowering branches in spring. It is very suitable for practicing Tai Chi. Moreover, in Yunnan, there is an almost lost 148 Royal Court Tai Chi. During the Qing dynasty, the Tai Chi Master Yang Lu Chan was recommended by Wangfu's steward Wang Lanting to teach Wang Quanfu. Wang Lanting worshipped under Yang Luzhen and became the disciple of Yang Lu Chan. Li Ruidong introduced the Yang Lu Chan as the teacher through Wang Lanting, followed by Yang Luchan and Wang Lanting to learn Tai Chi. Later Li Ruidong entered the palace and was promoted as a two-piece knife guard and spread Tai Chi in the palace; Li Ruidong and Zhang Hongsheng passed Tai Chi martial arts and medical ethics to Zhang Jinling's son Zhang Jinling. In 1939, after Zhang Jinling settled in Kunming from Beijing, he passed Tai Chi and other martial arts to Xu Shuizhang and then to Zhang Guoxiang. Zhang Jinling is the master of the nationalist party. Senior generals Bai Chongqi, Yang Sen, and Xue Yue, Xu Shuizhang followed General Chen Hao for two years. Although this Tai Chi was created by Yang Lu Chan, a Yang style Tai Chi master, the boxing and learning methods are very different from the general Yang style Tai Chi, which is now circulating in secret. If this Tai Chi is researched and developed, together with other Tai Chi schools, which is currently spreading in Kunming, it will be a great potential for Kunming to become a Tai Chi tourism and health resort in Southeast Asia and South Asia.

Other cities such as Hangzhou and Chengdu are also worthwhile to further develop Tai Chi tourism projects.

\section{Further enrichment of Tai Chi tourism products}

Although activities such as Tai Chi Study Tour and Tai Chi Tournament Tour have been carried out, the product form is still not rich enough. It is also necessary to further explore the integration points of Tai Chi and tourism, such as Tai Chi health tourism, Tai Chi rural tourism, and Tai Chi movie and television tours.

The term "health tourism" originated in Mexico and the United States in the 1930s. It is characterized by medical care and fitness activities. It caters to tourists seeking a relaxed, balanced living condition, avoiding crowded and polluted urban life. Taking into account the "health" and "tourism" eco- 
tourism forms. Tai Chi takes yin and yang theory as its core and integrates various functions such as supportive temperament, physical fitness, and combat and confrontation. It has been proved by many disciplines such as medicine, physiology, biochemistry, anatomy, psychology, and mechanics that Tai Chi is a preventive measure against old age sickness, high blood pressure, heart disease, lung disease, hepatitis, arthrosis, gastro-intestinal diseases, neurasthenia, and other chronic diseases. Tai Chi has good curative effects and has a good preventive effect on cancer. Almost all anticancer associations must practice Tai Chi. The development of Tai Chi health tourism should form cooperation with tourism resorts and nursing homes. There is an inn in Lijiang, Yunnan Province that has a host who likes Tai Chi. He teaches Tai Chi every morning to all the guests who stay in his inn, and this has greatly improves the occupancy rate of the inn.

In recent years, many urban residents, especially retired people, prefer to migrate to resorts with a suitable climate and beautiful scenery in summer and winter. During their vacations, local residents can carry out Tai Chi exercises and practice Tai Chi that can enrich the content of rural tourism. For example, in Luoyang Peninsula, Dali, Yunnan, in order to enrich tourism products, Tai Chi experts have set up five medical institutes to teach free Tai Chi lessons for guests on holiday on the island.

Since 2003, the Wenxian County Government of Henan Province has hired relevant agencies to compile and complete the "Tai Chi Sanctuary Chenjiagou Tourism Development Plan", and has invested more than CNY 6500 million to build Tai Chi ancestral shrine, ancestral halls, square frontages, East and West Memorial Arch, Yanglu Zen Boxing Department, Dong Dagou Boxing and Tai Chi Museum. Wenxian County Government and Hebei Tangshan Juxin Company signed an investment agreement and Juxin invested CNY 1.15 billion to build an international Tai Chi cultural exchange center and a Chinese Tai Chi health tourism base project. The movie and television industry took "Tai Chi 1", "Tai Chi 2" and series of television dramas in the background of Chenjiagou as a basis for Tai Chi movie and television tourism.

\section{Creating Tai Chi Performing Arts Program}

After large-scale performing arts programs such as "Impression of Yunnan", "Impression of Liu Sanjie", and "Impression of Lijiang" have been successfully combined with tourism, several large-scale martial arts creative performances featuring Shaolin martial arts culture such as "Inside and Outside the Monastery" "Shaolin in the Wind" and "Shaolin
Musical Grand Ceremony" have also achieved great success in integrating with tourism. These performing arts programs combined with the Shaolin martial arts culture are considered to be gradually changing the state of tourism in Henan, making Henan tourism nightlife no longer lack. Since its premiere in October 2007, the "Zen Shaolin Music Festival" has so far performed more than 700 performances. It has received nearly a million visitors at home and abroad, and has a box office income of more than CNY 60 million. The market prospects are good.

The Tai Chi Chart and the legendary stage drama Tai Chi Nebula show the beauty of Tai Chi. Taking the Tai Chi culture as an element, one can further develop more Tai Chi theme performing arts programs for tourists to watch. For example, 108 of the 148 Royal Court Tai Chi in Yunnan Province have been trained by the Tai Chi array, and it is currently almost official. If it is explored and created, it will be a very good performing arts program.

\section{E. Developing Forum-style Tai Chi Tourism Project}

In general international Tai Chi competitions, special forums have been set up, but the main event is competition, forum is the supplement. The forum-style Tai Chi tourism project mentioned here is based on forums, along with Tai Chi performances or competitions. For example, the business elites in the business community in Zhenghe Island used the Tai Chi Zen created by Jet Li and Ma Yun as an auxiliary performance project, which provided a relaxed and pleasant atmosphere for the forum and was also deeply loved by Tai Chi practitioners.

\section{CONCLUSION}

In short, Tai Chi tourism is a distinctive combination of sports, tourism, medical care, and production-supporting industries. If experts and scholars from various industries do some further studies, government promotes it actively, and enterprises participate in it actively, this new industry will certainly developed a lot.

\section{REFERENCES}

[1] Jingchu Net - Hubei Daily 2014-11-25, http://hb.qq.com/a/20141125/015495.htm

[2] Hubei Network 2018-1-31, http://news.hbtv.com.cn/p/1140564.html

[3] Shaolin Tai Chi "Fame and Unequal" Who came to give birth to the source of Tai Chi "hands, Source: Henan Party Construction Network http://www.hndjw.gov.cn/2009/10/15/13554.html 